\title{
Dangers of Bilateral Counterparty Risk: the fundamental impact of closeout conventions
}

\author{
Damiano Brigo \\ Dept. of Mathematics \\ King's College, London
}

\author{
Massimo Morini* \\ Banca IMI, Intesa-SanPaolo \\ and Bocconi University, Milan
}

First version March 13, 2010. This version November 1, 2018.

\begin{abstract}
We analyze the practical consequences of the bilateral counterparty risk adjustment. We point out that past literature assumes that, at the moment of the first default, a risk-free closeout amount will be used. We argue that the legal (ISDA) documentation suggests in many points that a substitution closeout should be used. This would take into account the risk of default of the survived party. We show how the bilateral counterparty risk adjustment changes strongly when a substitution closeout amount is considered. We model the two extreme cases of default independence and co-monotonicity, which highlight pros and cons of both risk free and substitution closeout formulations, and allow us to interpret the outcomes as dramatic consequences on default contagion. Finally, we analyze the situation when collateral is present.
\end{abstract}

AMS Classification Codes: $62 \mathrm{H} 20,91 \mathrm{~B} 70$

JEL Classification Codes: G12, G13

keywords: Bilateral Counterparty Risk, Credit Valuation Adjustment, Debit Valuation Adjustment, Closeout, Default Contagion, Bond Pricing, Default Correlation, Co-monotonic Defaults, Collateral Modeling.

\section{Introduction}

In this paper we analyze the practical consequences of the bilateral counterparty risk adjustment. We point out that past literature assumes that, at the moment of default, a risk-free closeout amount will be used. The closeout amount is the net present value of the residual deal which is computed when one party defaults, and that is used for default settlement. A risk-free closeout amount is a net present value that assumes that the surviving counterparty is default-free.

We argue here that the legal (ISDA) documentation on the settlement of a default does not confirm this assumption. Documentation suggests in many points that a 'substitution

\footnotetext{
${ }^{*}$ Corresponding author. This paper expresses the views of its authors and does not represent the institutions where the authors are working or have worked in the past. Such institutions, including Banca IMI, are not responsible for any use which may be made of this paper contents. We thank Giorgio Facchinetti, Marco Bianchetti, Luigi Cefis, Martin Baxter, Andrea Bugin, Vladimir Chorny, Josh Danziger, Igor Smirnov and other participants to the ICBI 2010 Global Derivatives and Risk Management Conference for helpful discussion. The remaining errors are our own. The authors would also like to give special thanks to Andrea Pallavicini and Andrea Prampolini for thoroughly and deeply discussing the research issues considered in this paper.
} 
closeout' should be used, namely a net present value of the residual deal as computed from a third market player that is eager to become the counterparty of the survived party for the residual deal, replacing the defaulted one. Such a substitution closeout can be different from a risk-free one. As a first difference, it would take into account the risk of default of the survived party, since a real market counterparty would never neglect it. The counterparty risk adjustments changes strongly when a substitution closeout amount is considered, and we show how to compute it. Also the effects at the moment of default of a company are very different under the two closeout conventions, with some dramatic consequences on default contagion. We show this in the following. In the current uncertainty about which closeout will be used in future defaults, our results should be considered carefully by the financial community, lest there are unpleasant surprises at the moment of default of a counterparty.

\section{Risk-free vs substitution closeout: Practical consequences}

We analyze the practical consequences of the two different ways of computing the closeout amount. We show on a simple derivative that the standard formula for bilateral counterparty risk adjustment, assuming risk-free closeout, is not consistent with the market practice on simple uncollateralized defaultable claims such as bonds or loans or options. In fact it causes the initial price of a deal to depend crucially on the risk of default of one party that has no future obligations in the deal. In case of a simple 'bond' or 'option' deal, it would make the price to depend on the risk of default of the 'lender', namely the party which has not future payments to make in the deal, such as the bond buyer or the option buyer. This feature is not consistent with current market practices and is counterintuitive. We show that this feature can be avoided by using the formula with substitution closeout that we introduce in this paper. In this sense, the substitution closeout formula that we introduce appears as the right choice to achieve consistency with market practice for uncollateralized deals.

However, the substitution closeout formula is not immune to problems. The main one regards the consistency with collateral computations for collateralized deals. The collateral is calculated computing a risk-free net present value. If the closeout is not risk-free how could the collateral amount and the closeout amount match at default in such a way that no losses are suffered by any party, as one would expect for collateralized deals? In the following we propose an explanation of how this can be the case even for a substitution closeout, but the issue would appear to be trivially solved under a risk-free closeout.

We also observe that, if we consider the above simple uncollateralized 'bond' deals under scenarios of perfect default dependency between the two counterparties, the risk-free closeout CVA is either the same as the substitution closeout CVA (this happens when the 'lender' has lower credit spreads, so that he never defaults first and we are in practice in a unilateral CVA) or it has a behaviour that could be considered not to be illogic, contrary to the substitution closeout. This second case happens when the 'lender' has higher credit spreads; in this case the risk-free closeout says that any bond should be treated as risk-free; this could be justified by the fact that the lender always defaults first so he will never be impacted by the default of the borrower. The situation changes when we consider counterparties with independent default risk, as we detail below.

\section{Forms of contagion implied by the two closeouts in different situations}

Another important point in comparing the two closeout formulations is understanding what happens upon default of one counterparty. In particular we focus on the case that shows the most striking differences with the unilateral case: the default of the counterparty with no future payments in the deal, the 'lender' in a synthetic loan transaction. Let us start with a quite relevant problem of the risk free closeout, having possibly destabilizing consequences.

When one of our counterparties defaults, we not only have losses if we are creditors of the 
defaulted company, we may have potentially large losses even if we are debtors, and these losses will be higher the higher our credit spreads. This fact is so central that it is worth repeating it: if we are debtors of a counterparty and this counterparty defaults, the accounting of bilateral counterparty risk given in previous literature prescribes that the default event of our creditor suddenly increases the amount of money that we have to pay. We illustrate this by sketching a practical example where we consider a bank that funded itself through a derivative. The bank finds out that default of the lender makes its liabilities grow from 579 million to 927 million just as an effect of the closeout assumption. This is a loss of 348 million on a funding deal of 1 billion notional. As we show in the following under the assumption of independence between lender and borrower, a substitution closeout would avoid this sudden loss and lead to a continuous counterparty risk mark-to-market for the borrower if the lender defaults.

Again, however, if we assume perfect dependence, there are additional considerations to make. We present a case where the default of the lender causes the liabilities of the borrower to decrease from $856 \mathrm{bn}$ to 0 if we consider the substitution closeout. This is a stylized representation of a not-impossible real world scenario where the default of one entity causes the spread of its counterparties to jump extremely high. This deflates their liabilities towards the defaulted company if we take into account their default probability in computing the closeout amount. This shows what is the main criticism that can be made to the substitution closeout: at the moment of default it protects the borrowers of the defaulted company, but in doing this it reduces the amount received by the creditors of the defaulted company (it reduces the recovery). Thus, the risk-free closeout increases the number of operators subject to contagion from a default, including parties that currently seem not to think they are exposed, and this is certainly a negative fact. On the other hand, it spreads the default losses on higher number of parties and reduces the classic contagion channel affecting creditors. For the creditors, this is certainly a positive fact.

It seems to us that this issue had never been studied thoroughly before, as confirmed by the fact that an absolute standard for the computation of the closeout amount seems not to exist among practitioners. Our mathematical and numerical results suggest that the issue should be considered carefully, both by practitioners and by regulators, since it has strong economic relevance, and in particular it can affect the contagion effect of a default event.

\section{Structure of the paper}

In Section 2 we present the formulas introduced by previous literature for counterparty risk adjustments. In Section 3 we analyze these formulas to clarify what they assume about the computation of the closeout amount, and we propose a closeout amount different from the risk-free one, namely the substitution closeout. Then in Sections 4 and 5 we analyze ISDA documentation. In Section 6 we perform the quantitative analysis of the problem. We first apply the alternative formulas to a very simple payoff, revealing that only the substitution closeout appears consistent with market practice for bonds and loans, while the risk-free closeout introduces even at initial time 0 a dependence on the risk of default of the party with no future obligation. The pattern of this unusual dependence is detailed numerically. Then we quantify the losses or the benefit, respectively, that a borrower would suffer in case of default of the lender when the substitution or risk-free closeouts are applied in the respective cases of independence and total default dependence. We have decided to consider only these limit cases - perfect dependence and independence - because they allow us to make claims which are as model-independent as possible, avoiding the use of 'black-box' copula models and associated concepts such as 'default correlation'.

In Section 7 we show that when the substitution closeout is used the combination of a defaultable deal and a collateral agreement may lead naturally to a default-free deal, as it should be. Finally in the Appendix we show a number of mathematical properties, such as symmetry, of the formula with substitution closeout that we introduce in this work. 


\section{The standard risk-free closeout bilateral formulas}

We consider two parties in a derivative transaction: $A$ (investor) and $B$ (counterparty). We call $\tau^{X}, R^{X}$ and $L^{X}=1-R^{X}$, respectively, the default time, the recovery and the loss given default of party $X$, with $X \in\{A, B\}$. The risk-free discount factor is

$$
D(t, T)=e^{-\int_{t}^{T} r(s) d s},
$$

where $r(t)$ is the risk-free short-rate. We define $\Pi_{A}(t, T)$ to be the discounted cashflows of the derivative from $t$ to $T$ seen from the point of view of $A$, with $\Pi_{B}(t, T)=-\Pi_{A}(t, T)$. The net present value of the derivative at $t$ is

$$
V_{A}^{0}(t):=\mathbb{E}_{t}\left[\Pi_{A}(t, T)\right],
$$

where $\mathbb{E}_{t}$ indicates the risk-neutral expectation based on market information up to time $t$. Notice that this, in general, includes default monitoring, i.e. the filtration at time $t$ includes $\left\{\tau_{X}>t\right\}$. We denote by $\mathbb{Q}_{t}$ the risk neutral probability measure conditional on the same information at time $t$.

The subscript $A$ indicates that this value is seen from the point of view of $A$, the superscript 0 indicates that we are considering both parties as default-free. Obviously, $V_{B}^{0}(t)=-V_{A}^{0}(t)$.

The early literature on counterparty risk adjustment, see for example Brigo and Masetti (2005), introduced 'unilateral risk of default'. Here only the default of counterparty $B$ is considered, while the investor $A$ is treated as default free. Under this assumption, the adjusted net present value to $A$ is

$$
\begin{aligned}
& V_{A}^{B}(t)=\mathbb{E}_{t}\left\{1_{\left\{\tau^{B}>T\right\}} \Pi_{A}(t, T)\right\}+ \\
& +\mathbb{E}_{t}\left\{1_{\left\{\tau^{B} \leq T\right\}}\left[\Pi_{A}\left(t, \tau^{B}\right)+D\left(t, \tau^{B}\right)\left(R^{B}\left(V_{A}^{0}\left(\tau^{B}\right)\right)^{+}-\left(-V_{A}^{0}\left(\tau^{B}\right)\right)^{+}\right)\right]\right\} \\
= & V_{A}^{0}(t)-\mathbb{E}_{t}\left[L^{B} 1_{\left\{\tau^{B} \leq T\right\}} D\left(t, \tau^{B}\right)\left(V_{A}^{0}\left(\tau^{B}\right)\right)^{+}\right]=: V_{A}^{0}(t)-\mathrm{CVA}_{A}(t) .
\end{aligned}
$$

The superscript $B$ indicates that this value allows for the risk of default of $B$. Notice that we always assume both parties to be alive at $t$. The approach is easily extended to the case when $B$ is treated as default-free, but the default of investor $A$ is taken into account. Now the adjusted net present value to $A$ is

$$
\begin{aligned}
& V_{A}^{A}(t)=\mathbb{E}_{t}\left\{1_{\left\{\tau^{A}>T\right\}} \Pi_{A}(t, T)\right\}+ \\
+ & \mathbb{E}_{t}\left\{1_{\left\{\tau^{A} \leq T\right\}}\left[\Pi_{A}\left(t, \tau^{A}\right)+D\left(t, \tau^{A}\right)\left(\left(V_{A}^{0}\left(\tau^{A}\right)\right)^{+}-R^{A}\left(-V_{A}^{0}\left(\tau^{A}\right)\right)^{+}\right)\right]\right\} \\
= & V_{A}^{0}(t)+\mathbb{E}_{t}\left[L^{A} 1_{\left\{\tau^{A} \leq T\right\}} D\left(t, \tau^{A}\right) \cdot\left(-V_{A}^{0}\left(\tau^{A}\right)\right)^{+}\right]=: V_{A}^{0}(t)+\operatorname{DVA}_{A}(t) .
\end{aligned}
$$

The extension to the most realistic case when both $A$ and $B$ can default is less trivial. This is called 'bilateral risk of default' and it is introduced for interest rate swaps in Bielecki and Rutkowski (2001), Picoult (2005) (where a simplified and approximated use of the indicators is adopted), Gregory (2009), Brigo and Capponi (2008), and Brigo Pallavicini and Papatheodorou (2009). In these previous works the net present value adjusted by the default probabilities of both parties is given by

$$
\begin{aligned}
& V_{A}(t)=\mathbb{E}_{t}\left\{1_{0} \Pi_{A}(t, T)\right\} \\
& +\mathbb{E}_{t}\left\{1_{A}\left[\Pi_{A}\left(t, \tau^{A}\right)+D\left(t, \tau^{A}\right)\left(\left(V_{A}^{0}\left(\tau^{A}\right)\right)^{+}-R^{A}\left(-V_{A}^{0}\left(\tau^{A}\right)\right)^{+}\right)\right]\right\} \\
& +\mathbb{E}_{t}\left\{1_{B}\left[\Pi_{A}\left(t, \tau^{B}\right)+D\left(t, \tau^{B}\right)\left(R^{B}\left(V_{A}^{0}\left(\tau^{B}\right)\right)^{+}-\left(-V_{A}^{0}\left(\tau^{B}\right)\right)^{+}\right)\right]\right\}
\end{aligned}
$$


where we use the following event indicators

$$
\begin{aligned}
1_{0} & =1_{\left\{T<\min \left(\tau^{A}, \tau^{B}\right)\right\}} \\
1_{A} & =1_{\left\{\tau^{A} \leq \min \left(T, \tau^{B}\right)\right\}} \\
1_{B} & =1-1_{A}-1_{0}=1_{\left\{\tau^{B}<\tau^{A}\right\}} 1_{\left\{\tau^{B} \leq T\right\}}
\end{aligned}
$$

Notice that

$$
V_{B}(t)=-V_{A}(t),
$$

thus this formula enjoys the symmetry property that one would expect. In the next section we analyze these formulas to understand what they implicitly assume about the settlement of a default event, and discuss the realism of such assumptions, and their practical consequences.

\section{The closeout problem: how much will be paid in case of a default, really?}

Knowing which default happens first is of fundamental importance for determining the actual payout. Thus it is convenient to rewrite formula (3) to make the order of the default events explicit.

We define 1 to be the first entity to default, and 2 to be the second one so that

$$
\tau^{1}=\min \left(\tau^{A}, \tau^{B}\right), \quad \tau^{2}=\max \left(\tau^{A}, \tau^{B}\right) .
$$

With these definitions the pricing formula (3) simplifies to

(5) $V_{A}(t)=\mathbb{E}_{t}\left\{1_{0} \Pi_{A}(t, T)\right\}$

$$
+\mathbb{E}_{t}\left\{\left(1_{B}-1_{A}\right)\left[\Pi_{2}\left(t, \tau^{1}\right)+D\left(t, \tau^{1}\right)\left(R^{1}\left(V_{2}^{0}\left(\tau^{1}\right)\right)^{+}-\left(-V_{2}^{0}\left(\tau^{1}\right)\right)^{+}\right)\right]\right\} .
$$

Let us compare this bilateral pricing formula with the price $V_{A}^{B}(t)$ given in (1). There the distinction between $\tau^{1}$ and $\tau^{2}$ is meaningless, since only the counterparty $B$ can default, so that

$$
\begin{aligned}
& \tau^{2}=\tau^{A}=+\infty \\
& \tau^{1}=\tau^{B}=: \tau
\end{aligned}
$$

Thus,

$$
\begin{aligned}
V_{A}^{B}(t)= & \mathbb{E}_{t}\left\{1_{\{\tau>T\}} \Pi_{A}(t, T)\right\} \\
& +\mathbb{E}_{t}\left\{1_{\{\tau \leq T\}}\left[\Pi_{A}(t, \tau)+D(t, \tau)\left(R^{1}\left(V_{A}^{0}(\tau)\right)^{+}-\left(-V_{A}^{0}(\tau)\right)^{+}\right)\right]\right\} .
\end{aligned}
$$

Now we introduce the main theme of this paper. All formulas for counterparty risk adjustment are based on precise assumptions on what happens when there is a default event. Let us start from the unilateral case (6). When the default of the counterparty happens before maturity (look at the part of the formula following the indicator of event $\{\tau \leq T\}$ ), the total payout is made of two parts: the cashflows received before default, $\Pi_{A}(t, \tau)$, and the present value of the payout at default time $\tau$. At $\tau$ the residual deal is marked-to-market. The mark-to-market of the residual deal at an early termination time is called closeout amount in the jargon of ISDA documentation. Here it is given by

$$
V_{A}^{0}(\tau)=-V_{B}^{0}(\tau)
$$


where the left-hand side is seen from the perspective of the investor and the right-hand side from the one of the counterparty. If the closeout amount is positive to $B$, which is the defaulting party, and negative to $A$, which has not defaulted, $A$ will pay this amount entirely to the liquidators of the counterparty. If the closeout amount is instead positive to $A$ and negative to $B$, the liquidators of the latter will pay to $A$ only a recovery fraction of the closeout amount. These provisions lead to the payout at default of (6), given by

$$
\left(R^{1}\left(V_{A}^{0}(\tau)\right)-\left(-V_{A}^{0}(\tau)\right)^{+}\right) .
$$

Notice that, as indicated by the superscript 0 , here the closeout amount $V_{A}^{0}(\tau)$ is computed treating the residual deal as a default-free deal. The reason for that is obvious. There are two parties $A$ and $B$, and party $A$ is supposed default-free so it will never default, while party $B$ has already defaulted and this is taken into account by the fact that, in case $V_{B}^{0}(\tau)<0, B$ will pay only a recovery fraction of the default-free closeout amount. This default-free closeout is also a substitution closeout, in the sense that, if $A$ wanted to substitute the defaulted deal with another one where the counterparty is default-free, the counterparty would ask $A$ to pay $V_{A}^{0}(\tau)$, a risk-free closeout since both parties are risk-free.

Now let us look at the pricing formula (5) for bilateral risk of default. The payout at default is now given by

$$
\left(1_{B}-1_{A}\right)\left[R^{1}\left(V_{2}^{0}\left(\tau^{1}\right)\right)^{+}-\left(-V_{2}^{0}\left(\tau^{1}\right)\right)^{+}\right]
$$

Here both $A$ and $B$ can default, and what matters is who defaults first. If the counterparty $B$ defaults first, then $1_{B}=1,1_{A}=0, \tau^{1}=\tau^{B}$ and we have the same payout as in the unilateral case of (7). If the investor $A$ defaults first, the payout is reversed: when the closeout amount is positive to the defaulted investor, this amount is received fully, while if it is negative only a recovery fraction will be paid to the counterparty, leading to

$$
\left[\left(V_{A}^{0}\left(\tau^{1}\right)\right)^{+}-R^{1}\left(-V_{A}^{0}\left(\tau^{1}\right)\right)^{+}\right] .
$$

Notice that, like in the unilateral case, also with bilateral risk of default the closeout amount is computed treating the residual deal as default-free

$$
V_{1}^{0}\left(\tau^{1}\right)=-V_{2}^{0}\left(\tau^{1}\right)
$$

We are again excluding the possibility of default of either party. Is this assumption as obviously justified here as it was in the unilateral case? Not quite. Only the assumption of ignoring the risk of default of 1 is justified obviously. In fact 1 has defaulted, and this is accounted for correctly by computing a closeout amount where there is no possibility of another default of 1 , and then, in case this amount is negative to 1 , assuming that 1 will pay only a recovery fraction of it. But the other party 2 has not defaulted, and now it is not true that it will never default in the future. There is a non-negligible probability that it defaults before the maturity $T$ of the residual deal. Thus it is unclear why the mark-to-market of a residual deal where one of the two parties has not yet defaulted and can default in the future should be treated as default free. Here the risk-free closeout amount (8) is not a substitution closeout. In fact if the survived party 2 wanted to substitute the defaulted deal with another one where the market counterparty is default free, the counterparty would ask 2 to pay not the opposite of (8) but

$$
V_{2}^{2}\left(\tau^{1}\right)=-V_{1}^{2}\left(\tau^{1}\right)
$$

because the market counterparty cannot ignore the default risk of party 2 from $\tau^{1}$ to the maturity $T$ of the residual deal. Thus the amount (9) will be called in the following substitution closeout amount. It is given by (11) when $1=B$ and by (2) when $1=A$. 
Allowing for this computation of the closeout amount, the pricing formula in case of bilateral counterparty risk becomes

$$
\begin{aligned}
& \hat{V}_{A}(t)=\mathbb{E}_{t}\left\{1_{0} \Pi_{A}(t, T)\right\} \\
& +\mathbb{E}_{t}\left\{1_{A}\left[\Pi_{A}\left(t, \tau^{A}\right)+D\left(t, \tau^{A}\right)\left(\left(V_{A}^{B}\left(\tau^{A}\right)\right)^{+}-R^{A}\left(-V_{A}^{B}\left(\tau^{A}\right)\right)^{+}\right)\right]\right\} \\
& +\mathbb{E}_{t}\left\{1_{B}\left[\Pi_{A}\left(t, \tau^{B}\right)+D\left(t, \tau^{B}\right)\left(R^{B}\left(V_{A}^{A}\left(\tau^{B}\right)\right)^{+}-\left(-V_{A}^{A}\left(\tau^{B}\right)\right)^{+}\right)\right]\right\} .
\end{aligned}
$$

In the rest of the paper we analyze this formula under two points of view. First, we want to understand if it is more appropriate than formula (3) used in the previous literature. For this analysis we consider

1) the ISDA documentation on derivatives, to understand the legal prescriptions and the financial rationale that should be followed in computing the closeout amount

2 ) which one between the substitution closeout amount assumed by (10) and the risk-free one assumed by (3) is simpler to be applied in case of a real default.

3) the financial effects of using (10) rather than (3), to see which one minimizes the default contagion in case of default, which one is more consistent with market standards on consolidated financial products and which one fits better with market practices to minimize default risk such as CSA collateral agreements.

One observation is in order about this plan of analysis. The reader may think that the first step to take is to ask experienced practitioners which approach is applied in practice for the computation of closeout. We have done this (and we thank for that in particular the participants to Global Derivatives 2010 in Paris) and surprisingly enough we have found different opinions. This variety of opinions can be related to the fact that the ISDA documentation had given a (relatively) open definition of closeout amount, and more importantly to the fact that full awareness of the importance of CVA and DVA has arisen just after Lehman's default, the last important default that may work as a benchmark. At that time "Libor discounting" was mainly used for closeout, and this is somehow in-between a risk-free discounting and a discounting taking full consideration of the risk of default of the remaining party.

Then, in the appendix, we analyze the new formula (10) under a mathematical point of view. We first show that it enjoys the symmetry property $\hat{V}_{A}(t)=-\hat{V}_{B}(t)$ as well. Then we show that is equivalent to

$$
\begin{aligned}
& \hat{V}_{A}(t)=\mathbb{E}_{t}\left\{\Pi_{A}(t, T)\right\} \\
& +\mathbb{E}_{t}\left\{1 _ { 1 } \left[D ( t , \tau ^ { A } ) \left(-\mathbb{E}_{\tau^{A}}\left[L^{B} 1_{\left\{\tau^{B} \leq T\right\}} D\left(\tau^{A}, \tau^{B}\right) \cdot\left(V_{A}^{0}\left(\tau^{B}\right)\right)^{+}\right]\right.\right.\right. \\
& \left.\left.\left.+\left(1-R^{A}\right)\left(-V_{A}^{0}\left(\tau^{A}\right)+\mathbb{E}_{\tau^{A}}\left[L^{B} 1_{\left\{\tau^{B} \leq T\right\}} D\left(\tau^{A}, \tau^{B}\right) \cdot\left(V_{A}^{0}\left(\tau^{B}\right)\right)^{+}\right]\right)^{+}\right)\right]\right\} \\
& +\mathbb{E}_{t}\left\{1 _ { 2 } \left[D ( t , \tau ^ { B } ) \left(\mathbb{E}_{\tau^{B}}\left[L^{A} 1_{\left\{\tau^{A} \leq T\right\}} D\left(\tau^{B}, \tau^{A}\right) \cdot\left(-V_{A}^{0}\left(\tau^{A}\right)\right)^{+}\right]\right.\right.\right. \\
& \left.\left.\left.-\left(1-R^{B}\right)\left(V_{A}^{0}\left(\tau^{B}\right)+\mathbb{E}_{\tau^{B}}\left[L^{A} 1_{\left\{\tau^{A} \leq T\right\}} D\left(\tau^{B}, \tau^{A}\right) \cdot\left(-V_{A}^{0}\left(\tau^{A}\right)\right)^{+}\right]\right)^{+}\right)\right]\right\},
\end{aligned}
$$

and that, using the definitions in (4) and recalling that $V_{B}^{0}(t)=-V_{A}^{0}(t)$, it can be simplified in

$$
\begin{aligned}
& \hat{V}_{A}(t)=\mathbb{E}_{t}\left\{1_{0} \Pi_{A}(t, T)\right\} \\
& +\mathbb{E}_{t}\left\{\left(1_{B}-1_{A}\right)\left[\Pi_{2}\left(t, \tau^{1}\right)+D\left(t, \tau^{1}\right)\left(R^{1}\left(V_{2}^{2}\left(\tau^{1}\right)\right)^{+}-\left(V_{1}^{2}\left(\tau^{1}\right)\right)^{+}\right)\right]\right\} .
\end{aligned}
$$




\section{A 'legal analysis': the ISDA closeout amount protocol}

The document that should set a standard for the computation of the close-out amount in case of a default event is the ISDA (2009) Close-out Amount Protocol. Nowhere in this document one finds a precise formula for the computation of the close-out amount, however one can find there practical principles that can shed some light on the issue we are considering. We read at page 13 that "If the Early Termination Date results from an Event of Default", this early termination will be settled by the transfer of "the Close-out Amount or Close-out Amounts (whether positive or negative) determined by the Non-defaulting Party". The non-defaulting party that determines the closeout amount is party 2 in our notation. Then at page 15 we have the following prescription: "In determining a Close-out Amount, the Determining Party may consider any relevant information, including, without limitation, one or more of the following types of information: (i) quotations (either firm or indicative) for replacement transactions supplied by one or more third parties that may take into account the creditworthiness of the Determining Party at the time the quotation is provided". This is in contrast with the defaultfree closeout amount $V_{2}^{0}\left(\tau^{1}\right)$ prescribed by the classic formula, and seems instead consistent with the substitution-cost closeout amount $V_{2}^{2}\left(\tau^{1}\right)$ prescribed by the formula given in this paper, that includes the risk of default of the survived party 2. The ISDA documentation is not so strict to make this a binding prescription - the document speaks of a determining party that may take into account its own creditworthiness. Thus the risk-free closeout amount is not excluded.

Various other points in the documentation confirm that a substitution closeout is more likely. One of the clearest is in the Market Review of OTC Derivative Bilateral Collateralization Practices, published by ISDA on March 1, 2010, that says: "Upon default close-out, valuations will in many circumstances reflect the replacement cost of transactions calculated at the terminating party's bid or offer side of the market, and will often take into account the credit-worthiness of the terminating party".

The same document, however, points out that this substitution closeout risks being a problem for collateralized derivatives, since it seems at odds with the computation of collateral amount: "However, it should be noted that Exposure is calculated at mid-market levels so as not to penalize one party or the other. As a result of this, the amount of collateral held to secure Exposure may be more or less than the termination payment determined upon a close-out". We analyze this issue in detail in Section 7

\section{Risk-free vs substitution closeout: what is simpler to compute?}

The above analysis shows a financial rationale in the ISDA documents in favor of a substitution closeout, but it also shows that the same documents leave room for other solutions. There is one point in strong favor of a risk-free closeout: the simplicity of computation, since it does not require an assessment of the risk of default of the survived party. In the settlement of a default, if a risk-free closeout is used, then all contracts with the same payoff would have the same closeout value, irrespectively of the counterparty, which is an important simplification. This risk-free amount would not be difficult to compute in the market because it would correspond to the mark-to-market of a deal equal to the residual deal but for the fact of being collateralized. Of course some parties could find it unfair. In the end the defaulted deal was not collateralized, and at inception it had different prices for different parties because of the different default risks of the parties. Some may not accept now a unique 'collateralized' closeout amount. In spite of this, the homogeneity of the risk-free closeout amount is a strong point in favor of this solution, maybe just as a simpler approximation to the substitution closeout, if the error turns out to be not too big. 
The above considerations do not appear sufficient to clarify our minds and settle the matter without a more quantitative analysis. We perform such analysis in the following. Since we are dealing with a matter that would affect even the simplest derivative in case of default, we find an analysis with very simple - albeit not unrealistic - models and transactions to be much more instructive than the application of some elaborate model to a complex derivative, that would just risk increasing the confusion. Once we have sorted out the basic case, we can think of addressing more complex payouts in future work.

\section{A quantitative analysis: pricing a bond payoff}

We perform a mathematical and numerical analysis of the consequences of the two approaches. We consider a contract where party $B$ enters at time 0 into the commitment to pay a unit of money at time $T$ to party $A$. This claim has exactly the same payoff as the prototypical zero-coupon bond or loan of the textbooks on finance. Party $B$ is the borrower or bond issuer, and $A$ is the lender or bond holder. This payoff is convenient for our purposes because there is a consolidated standard on how to price it. We will see which one between risk-free and substitution liquidation is consistent with the practice developed in the loan and bond markets.

The comparison with the bond market is particularly interesting for a further reason. When bilateral counterparty risk was first introduced, there was some discussion in the market about one consequence of it: when a bank includes its own risk of default in the pricing of a deal, it can actually book a profit when there is an increase of its credit spreads. This is a rather bizarre fact. However supporters of this approach pointed out that this already happens for banks with reference to bond issuances. Banks have the so-called fair value option, namely the possibility to account for issued bonds in their balance-sheets at mark-to-market. When this is done, the bond liabilities decrease in value when credit spreads increase, and the bank can mark a profit. This consistency with the treatment of bonds has contributed to make the DVA more accepted in the market. Below we will show some practical effects of bilateral counterparty risk adjustments under risk-free closeout or substitution closeout. By observing these effects on a bond-like payoff, we are using the payoff that has already been the main reference for understanding the appropriateness of counterparty risk adjustments.

\subsection{Pricing a bond under risk-free or substitution closeout}

In the following we take deterministic interest rates.

\subsubsection{The unilateral case}

First we evaluate this 'derivative bond' deal using unilateral formulas, namely considering only the default of the borrower counterparty $B$ (here the default of $A$ is neglected from the payout irrespectively of the fact that it may default or not). We apply (11) to this payoff, getting

$$
\begin{aligned}
(11) V_{A}^{B}(t) & =\mathbb{E}_{t}\left\{1_{\left\{\tau^{B}>T\right\}} e^{-\int_{t}^{T} r(s) d s}\right\}+\mathbb{E}_{t}\left\{1_{\left\{\tau^{B} \leq T\right\}}\left[e^{-\int_{t}^{\tau^{B}} r(s) d s} R^{B}\left(e^{-\int_{\tau^{B}}^{T} r(s) d s}\right)^{+}\right]\right\} \\
& =e^{-\int_{t}^{T} r(s) d s} \mathbb{E}_{t}\left[1_{\left\{\tau^{B}>T\right\}}\right]+R^{B} e^{-\int_{t}^{T} r(s) d s} \mathbb{E}_{t}\left[1_{\left\{\tau^{B} \leq T\right\}}\right]
\end{aligned}
$$

This is the standard formula for the pricing of a defaultable bond or loan. We have

$$
V_{A}^{B}(0)=e^{-\int_{0}^{T} r(s) d s} \mathbb{Q}\left(\tau^{B}>T\right)+R^{B} e^{-\int_{0}^{T} r(s) d s} \mathbb{Q}\left(\tau^{B} \leq T\right),
$$

which says that the price of a defaultable bond equals the price of a default-free bond multiplied by the survival probability of the issuer, plus a recovery part received when the issuer defaults. 
By applying (2) to this payoff we can compute easily also $V_{A}^{A}(t)$, the value when only the default of the lender $A$ is taken into account. We have

$V_{A}^{A}(t)=\mathbb{E}_{t}\left\{1_{\left\{\tau^{A}>T\right\}} e^{-\int_{t}^{T} r(s) d s}\right\}+\mathbb{E}_{t}\left\{1_{\left\{\tau^{A} \leq T\right\}} e^{-\int_{t}^{\tau^{A}} r(s) d s}\left(e^{-\int_{\tau A}^{T} r(s) d s}\right)^{+}\right\}=e^{-\int_{t}^{T} r(s) d s}$.

We have obtained the price of a risk-free bond, thus the formula says that in a loan or bond what matters is the risk of default of the borrower. If we consider only risk of default for the lender, and not default-risk of the borrower, we get just the price of a default-free loan or bond. We have no influence of the risk of default of a party, the lender, that in this contract has no future obligations. Both formula (11) and (12) are in line with market practice.

\subsubsection{The bilateral case with substitution closeout}

Now we price the deal considering the default risk of both parties, and assuming first a substitution closeout. We apply formula (10) introduced in this paper, putting (11) and (12) into this formula. We get

$$
\begin{aligned}
& \hat{V}_{A}(t)=\mathbb{E}_{t}\left\{1_{0} e^{-\int_{t}^{T} r(s) d s}\right\} \\
& +\mathbb{E}_{t}\left\{1_{A}\left[e^{-\int_{t}^{\tau^{A}} r(s) d s}\left(e^{-\int_{\tau^{A}}^{T} r(s) d s} \mathbb{E}_{\tau^{A}}\left[1_{\left\{\tau^{B}>T\right\}}\right]+R^{B} e^{-\int_{\tau^{A}}^{T} r(s) d s} \mathbb{E}_{\tau^{A}}\left[1_{\left\{\tau^{B} \leq T\right\}}\right]\right)\right]\right\} \\
& +\mathbb{E}_{t}\left\{1_{B}\left[e^{-\int_{t}^{\tau^{B}} r(s) d s} R^{B}\left(e^{-\int_{\tau^{B}}^{T} r(s) d s}\right)^{+}\right]\right\} \\
& =\mathbb{E}_{t}\left\{1_{0} e^{-\int_{t}^{T} r(s) d s}\right\} \\
& +\mathbb{E}_{t}\left\{1_{A}\left[e^{-\int_{t}^{T} r(s) d s} \mathbb{E}_{\tau^{A}}\left[1_{\left\{\tau^{B}>T\right\}}\right]+R^{B} e^{-\int_{t}^{T} r(s) d s} \mathbb{E}_{\tau^{A}}\left[1_{\left\{\tau^{B} \leq T\right\}}\right]\right\}\right. \\
& +\mathbb{E}_{t}\left\{1_{B}\left[R^{B} e^{-\int_{t}^{T} r(s) d s}\right]\right\}
\end{aligned}
$$

Notice that

$$
1_{A}=1_{\left\{\tau^{A} \leq \min \left(T, \tau^{B}\right)\right\}}
$$

is $\tau^{A}$-measurable, namely it is known at $\tau^{A}$, so that the second one of the three terms of $\hat{V}_{A}(t)$ above can be rewritten as

$$
\begin{aligned}
& \mathbb{E}_{t}\left\{1_{A}\left[e^{-\int_{t}^{T} r(s) d s} \mathbb{E}_{\tau^{A}}\left[1_{\left\{\tau^{B}>T\right\}}\right]+R^{B} e^{-\int_{t}^{T} r(s) d s} \mathbb{E}_{\tau^{A}}\left[1_{\left\{\tau^{B} \leq T\right\}}\right]\right]\right\} \\
= & e^{-\int_{t}^{T} r(s) d s} \mathbb{E}_{t}\left[\mathbb{E}_{\tau^{A}}\left[1_{A} 1_{\left\{\tau^{B}>T\right\}}\right]\right]+R^{B} e^{-\int_{t}^{T} r(s) d s} \mathbb{E}_{t}\left[\mathbb{E}_{\tau^{A}}\left[1_{A} 1_{\left\{\tau^{B} \leq T\right\}}\right]\right] \\
= & e^{-\int_{t}^{T} r(s) d s} \mathbb{E}_{t}\left[1_{A} 1_{\left\{\tau^{B}>T\right\}}\right]+R^{B} e^{-\int_{t}^{T} r(s) d s} \mathbb{E}_{t}\left[1_{A} 1_{\left\{\tau^{B} \leq T\right\}}\right]
\end{aligned}
$$

where in the last passage we have used the law of iterated expectations. Now in $\hat{V}_{A}(t)$ we can factor together the terms that are multiplied by $R^{B}$ and those that are not, getting

$$
\begin{aligned}
\hat{V}_{A}(t)= & e^{-\int_{t}^{T} r(s) d s} \mathbb{E}_{t}\left[1_{0}\right]+e^{-\int_{t}^{T} r(s) d s} \mathbb{E}_{t}\left[1_{A} 1_{\left\{\tau^{B}>T\right\}}\right] \\
& +R^{B} e^{-\int_{t}^{T} r(s) d s} \mathbb{E}_{t}\left[1_{A} 1_{\left\{\tau^{B} \leq T\right\}}\right]+R^{B} e^{-\int_{t}^{T} r(s) d s} \mathbb{E}_{t}\left[1_{B}\right] \\
= & e^{-\int_{t}^{T} r(s) d s} \mathbb{E}_{t}\left[1_{0}+1_{A} 1_{\left\{\tau^{B}>T\right\}}\right] \\
& +R^{B} e^{-\int_{t}^{T} r(s) d s} \mathbb{E}_{t}\left[1_{A} 1_{\left\{\tau^{B} \leq T\right\}}+1_{B}\right]
\end{aligned}
$$

Now we concentrate on the indicators. It is easy to see that

$$
\begin{aligned}
1_{A} 1_{\left\{\tau^{B}>T\right\}} & =1_{\left\{\tau^{A} \leq \min \left(T, \tau^{B}\right)\right\}} 1_{\left\{\tau^{B}>T\right\}}=1_{\left\{\tau^{A} \leq T\right\}} 1_{\left\{\tau^{B}>T\right\}} \\
1_{A} 1_{\left\{\tau^{B} \leq T\right\}} & =1_{\left\{\tau^{A} \leq \min \left(T, \tau^{B}\right)\right\}} 1_{\left\{\tau^{B} \leq T\right\}}=1_{\left\{\tau^{A} \leq \tau^{B}\right\}} 1_{\left\{\tau^{B} \leq T\right\}} \\
1_{0} & =1_{\left\{T<\min \left(\tau^{A}, \tau^{B}\right)\right\}}=1_{\left\{T<\tau^{A}\right\}} 1_{\left\{T<\tau^{B}\right\}}
\end{aligned}
$$


leading to

$$
\begin{aligned}
\hat{V}_{A}(t)= & e^{-\int_{t}^{T} r(s) d s} \mathbb{E}_{t}\left[1_{\left\{T<\tau^{A}\right\}} 1_{\left\{T<\tau^{B}\right\}}+1_{\left\{\tau^{A} \leq T\right\}} 1_{\left\{\tau^{B}>T\right\}}\right] \\
& +R^{B} e^{-\int_{t}^{T} r(s) d s} \mathbb{E}_{t}\left[1_{\left\{\tau^{A} \leq \tau^{B}\right\}} 1_{\left\{\tau^{B} \leq T\right\}}+1_{\left\{\tau^{B}<\tau^{A}\right\}} 1_{\left\{\tau^{B} \leq T\right\}}\right] .
\end{aligned}
$$

Since $1_{\left\{T<\tau^{A}\right\}}+1_{\left\{\tau^{A} \leq T\right\}}=1_{\left\{\tau^{A} \leq \tau^{B}\right\}}+1_{\left\{\tau^{B}<\tau^{A}\right\}}=1$, we have

$$
\begin{aligned}
\hat{V}_{A}(t) & =e^{-\int_{t}^{T} r(s) d s} \mathbb{E}_{t}\left[1_{\left\{\tau^{B}>T\right\}}\right]+R^{B} e^{-\int_{t}^{T} r(s) d s} \mathbb{E}_{t}\left[1_{\left\{\tau^{B} \leq T\right\}}\right] \\
& =e^{-\int_{t}^{T} r(s) d s}\left[\mathbb{Q}_{t}\left(\tau^{B}>T\right)+R^{B} \mathbb{Q}_{t}\left(\tau^{B} \leq T\right)\right] .
\end{aligned}
$$

In spite of the somewhat lengthy computations, we have found a very simple and reasonable result. We have $\hat{V}_{A}(t)=V_{A}^{B}(t)$, and we have that the risk of default of the bond-holder in a bond, or the lender in a loan, does not influence the value of the contract.

Remark 1. We point out that in the above formula for $\hat{V}_{A}(t)$ there can be dependence from the risk of default of the lender through the terms $\mathbb{Q}_{t}\left(\tau^{B}>T\right)$ and $\mathbb{Q}_{t}\left(\tau^{B} \leq T\right)$ when the formula is evaluated at a future time $t>0$. In fact in some models terms such as $\mathbb{Q}_{t}\left(\tau^{B}>T\right)$ do depend on the the risk of default of $A$. For example, when using a copula model, at times $t>0$ the fact that a correlated counterparty has defaulted or not by $t$ changes the default probability of a counterparty still alive, since dependency is set on unobservable latent variables (the exponential triggers), about which one can get information by observing if correlated companies have defaulted or not. In other models such as the structural first passage model of Black and Cox (1976) or the multivariate exponential Marshall Olkin (1967) model this does not happen. We point out that however also within models, like copulas, where terms such as $\mathbb{Q}_{t}\left(\tau^{B}>T\right)$ do depend on the the risk of default of $A$, pricing is always made assuming $t=0$, where even in these models there is independence from the the risk of default of $A$. The situation is different instead when in a copula we make a forward valuation where we need to assume $t>0$, as we do in the following when we asses the value of a deal just before and just after a default. There we show how conditioning on information about $A$ modifies $\mathbb{Q}_{t}\left(\tau^{B}>T\right)$.

We can conclude that, although bilateral risk of default matters in general for bilateral contracts, as confirmed by the fact that $\hat{V}_{A}(0)$ is in general much more complex that $V_{A}^{B}(0)$, with substitution closeout we have that when the contract has no future obligations for a party $A$ the risk of default of $A$ does not influence the price. Only the risk of default of the bond-issuer or borrower matters for valuation. This result is also in line with market practice 1

\subsubsection{The bilateral case with risk-free closeout}

Now we apply instead the formula (3) that assumes a risk-free closeout. Since $V_{A}^{0}(\tau)=$ $e^{-\int_{\tau}^{T} r(s) d s}$, we have

$$
\begin{aligned}
& V_{A}(t)=\mathbb{E}_{t}\left\{1_{0} e^{-\int_{t}^{T} r(s) d s}\right\}+\mathbb{E}_{t}\left\{1_{A}\left[e^{-\int_{t}^{\tau^{A}} r(s) d s}\left(e^{-\int_{\tau A}^{T} r(s) d s}\right)^{+}\right]\right\} \\
& +\mathbb{E}_{t}\left\{1_{B}\left[e^{-\int_{t}^{\tau^{B}} r(s) d s} R^{B}\left(e^{-\int_{\tau B}^{T} r(s) d s}\right)^{+}\right]\right\} \\
& =e^{-\int_{t}^{T} r(s) d s} \mathbb{E}_{t}\left[1_{0}+1_{A}\right]+e^{-\int_{t}^{T} r(s) d s} R^{B} \mathbb{E}_{t}\left[1_{B}\right] .
\end{aligned}
$$

\footnotetext{
${ }^{1}$ The reader may think that the risk of default of the lender could in practice influence the price of a bond or loan through its effect on the cost of funding for the lender. See Morini and Prampolini (2010) for a discussion on this. However, when liquidity costs are note considered, like in this paper, this effect does not exist and, like in classic bond pricing, one expects the risk of default of the holder not to influence the price of a bond.
} 
We can write

(14) $V_{A}(t)=e^{-\int_{t}^{T} r(s) d s} \mathbb{Q}_{t}\left[T<\min \left(\tau^{A}, \tau^{B}\right) \cup \tau^{A} \leq \min \left(T, \tau^{B}\right)\right]+e^{-\int_{t}^{T} r(s) d s} R^{B} \mathbb{Q}_{t}\left[\tau^{B}<\tau^{A} \cap \tau^{B} \leq T\right]$.

Playing with indicators one can also obtain the alternative expressions

$$
\text { (15) } \begin{aligned}
V_{A}(t) & =e^{-\int_{t}^{T} r(s) d s}\left(\mathbb{Q}_{t}\left[\tau^{B}>\min \left(\tau^{A}, T\right)\right]+R^{B} \mathbb{Q}_{t}\left[\tau^{B}<\min \left(\tau^{A}, T\right)\right]\right) \\
& =e^{-\int_{t}^{T} r(s) d s}\left(\mathbb{Q}_{t}\left[\tau^{B}>T\right]+\mathbb{Q}_{t}\left[\tau^{A}<\tau^{B}<T\right]+R^{B} \mathbb{Q}_{t}\left[\tau^{B}<\min \left(\tau^{A}, T\right)\right]\right)
\end{aligned}
$$

\subsubsection{Comparing the two closeout formulations}

Reaching this result for $V_{A}$ applied to a 'derivative bond' has been far easier than reaching the analogous one for $\hat{V}_{A}$, but the result looks more complex. In fact it introduces a dependence on the risk of default of the lender even at time 0 , and on the exact individuation of the first default, that was not there in $\hat{V}_{A}$. This is in contrast with the market practice in the bond or loan markets. In particular, let us compare $V_{A}(t)$ with $\hat{V}_{A}(t)$. Since

$$
\begin{aligned}
1_{0}+1_{A} & =1_{\left\{\tau^{B}>T\right\}} 1_{\left\{\tau^{A}>T\right\}}+1_{\left\{\tau^{B} \geq \tau^{A}\right\}} 1_{\left\{\tau^{A} \leq T\right\}} \\
& \geq \\
1_{\left\{\tau^{B}>T\right\}} & =1_{\left\{\tau^{B}>T\right\}} 1_{\left\{\tau^{A}>T\right\}}+1_{\left\{\tau^{B}>T\right\}} 1_{\left\{\tau^{A} \leq T\right\}}
\end{aligned}
$$

and $R^{B} \leq 1$, we have

$$
V_{A}(t) \geq \hat{V}_{A}(t)=V_{A}^{B}(t) .
$$

Thus a risk-free liquidation increases the value of a 'derivative bond' to the bond holder compared to the value that a bond has in the market practice. Symmetrically, the value is reduced to the bond issuer, and this reduction is an increasing function of the default risk of the bond holder. This confirms that a substitution closeout guarantees the borrower, the party which has payment to do in the future, making its risk of default the only relevant variable that determines the value of a bond or loan. This is a crucial feature for the stability of a debt market, but it has implications that can appear paradoxical. An example is in the following.

\subsubsection{A special case with comonotonic defaults}

Let us consider again the case of a risk free zero coupon bond, and assume the total default dependence case, as represented by two co-monotonic default times. We assume co-monotonicity as

$$
\tau^{A}=\psi\left(\tau^{B}\right)
$$

for a deterministic and strictly increasing function $\psi$. Let us further limit ourselves to situations where the lender is riskier than the borrower in terms of probability of default. This is represented by assuming that $\psi(x)<x$, so that $\tau^{A}<\tau^{B}$ in all scenarios. This is clearly a very extreme case; some example may happen if $B$ is somehow a subsidiary of $A$, although in a real-world case a default time will never be exactly a deterministic function of another default time (unless we consider the case of simultaneous defaults, that in fact is what usually holds for subsidiaries). Some models based on a physical explanation of default dependency, such as structural models or the multivariate exponential Marshall Olkin (1967) model we use in the following, do not even admit comonotonicity outside the case of simultaneous default. However this is a scenario admitted by other common models, like a bivariate default intensity model where default intensities of $A$ and $B$ satisfy $\lambda^{A}>\lambda^{B}$ and where the two default time exponential triggers are connected by the co-monotonic copula, or equivalently by a Gaussian copula with correlation 1 . Here default of a company triggers the default of a second one in a fully predictable way, but the default of the second one may happen any number of years 
after the default of the first one. The authors of this paper have pointed out in Brigo and Chourdakis (2008) and in Morini (2009) the risks of using models that admit comonotonic but not simultaneous default times, a feature with scarce financial meaning that can lead to misleading results. Here however we do not use this example because we think it is realistic, but to point out some extreme consequences of the choice about the closeout.

In the above setup we have that the risk free closeout formula (15) yields

$$
V_{A}(t)=e^{-\int_{t}^{T} r(s) d s}
$$

whereas the substitution closeout formula yields

$$
\hat{V}_{A}(t)=e^{-\int_{t}^{T} r(s) d s} \mathbb{Q}_{t}\left(\tau^{B}>T\right) .
$$

We are in a situation where whenever there is default, $A$ defaults always first. As a consequence, $A$ will never be impacted by $B$ 's default, and one could expect then the price of the bond to $A$ not to depend on the default risk of $B$. While this happens with the risk-free closeout, this does not happen with the substitution closeout, that maintains dependence on default risk of $B$. We have to say, however, that although in this stylized example it would make sense for the bond price not to depend on the default probability of $B$, this does not happen in the market, where the bond price remains $\hat{V}_{A}(t)$ regardless of default dependence issues, making the price of bonds the same for all buyers.

\subsection{A simple two-name credit model}

To quantify the size of the above difference, and to analyze numerically the practical effect of either assumption on closeout, we need to have a model for the default times of our two names. Consistently with the purpose of keeping complexity as low as possible since we are dealing with very fundamental issues, we will use the simplest bivariate extension of the standard single name credit model. Like in the single name market credit model, we assume that the default time of the name $X, X \in\{A, B\}$, is exponentially distributed. We take a flat default intensity $\lambda_{X}$, so that the survival probability is

$$
\mathbb{Q}\left(\tau_{X}>T\right)=e^{-\lambda_{X} T},
$$

and for consistency also $r(s)$ is taken flat so that the default-free bond is

$$
P_{T}=e^{-r T} .
$$

As baseline hypotheses, we take the two extreme scenarios, namely one scenario where the variables $\tau^{A}$ and $\tau^{B}$ are independent, and a second one where they are co-monotonic. We start with the independent case. It is well known that in this case $\min \left(\tau^{A}, \tau^{B}\right)$ is also exponentially distributed of parameter $\lambda_{A}+\lambda_{B}$, since

$$
\begin{aligned}
\mathbb{Q}\left(\min \left(\tau^{A}, \tau^{B}\right)>T\right) & =\mathbb{Q}\left(\tau^{A}>T\right) \mathbb{Q}\left(\tau^{B}>T\right) \\
& =e^{-\left(\lambda_{A}+\lambda_{B}\right) T}
\end{aligned}
$$

It is easy to compute the terms needed to apply Formula (14). First, one has

$$
\mathbb{Q}\left(\tau^{A}<\min \left(\tau^{B}, T\right)\right)=\frac{\lambda_{A}}{\lambda_{A}+\lambda_{B}}\left(1-e^{-\left(\lambda_{A}+\lambda_{B}\right) T}\right),
$$

as one can easily show by solving the integral

$$
\mathbb{Q}\left(\left\{\tau^{B}>\tau^{A}\right\} \cap\left\{T>\tau^{A}\right\}\right)=\int_{0}^{T} \mathbb{Q}\left(\tau^{B}>t\right) \mathbb{Q}\left(\tau^{A} \in d t\right)=\int_{0}^{T} e^{-\lambda_{B} t} \lambda_{A} e^{-\lambda_{A} t} d t
$$


We have also the special case

$$
\mathbb{Q}\left(\tau^{A}<\tau^{B}\right)=\frac{\lambda_{A}}{\lambda_{A}+\lambda_{B}}
$$

which is obtained as a limit case of the earlier expression when $T \uparrow \infty$. We have all we need for computing

$$
\begin{aligned}
& V_{A}(t)=e^{-r T}\left\{\mathbb{Q}\left[T<\min \left(\tau^{A}, \tau^{B}\right)\right]+\mathbb{Q}\left[\tau^{A}<\min \left(T, \tau^{B}\right)\right]+R^{B} \mathbb{Q}\left[\tau^{B}<\min \left(\tau^{A}, T\right)\right]\right\}= \\
& (16)=e^{-r T}\left\{e^{-\left(\lambda_{A}+\lambda_{B}\right) T}+\frac{\lambda_{A}}{\lambda_{A}+\lambda_{B}}\left(1-e^{-\left(\lambda_{A}+\lambda_{B}\right) T}\right)+R^{B} \frac{\lambda_{B}}{\lambda_{A}+\lambda_{B}}\left(1-e^{-\left(\lambda_{A}+\lambda_{B}\right) T}\right)\right\}
\end{aligned}
$$

Notice that in our model the probability of events such as $\tau^{A}=T$ or $\tau^{A}=\tau^{B}$ is zero.

In the comonotonic default case, we assume that

$$
\tau^{A}=\xi / \lambda_{A}, \quad \tau^{B}=\xi / \lambda_{B},
$$

with $\xi$ standard exponential random variable. It follows that

$$
\tau^{A}=\frac{\lambda_{B}}{\lambda_{A}} \tau^{B}
$$

In this case it will be easy to compute the terms appearing in (14). We begin with the case where $\lambda_{B}>\lambda_{A}$. Now $\tau^{B}$ always happens first, so we have

$$
V_{A}(t)=e^{-\int_{t}^{T} r(s) d s}\left(\mathbb{Q}_{t}\left[\tau^{B}>T\right]+R^{B} \mathbb{Q}_{t}\left[\tau^{B}<T\right]\right)=\hat{V}_{A}(t) .
$$

Hence in this case risk free closeout and substitution closeout agree. Furthermore, here it does not make sense to ask what happens at the default time of the lender, because the borrower defaults first and closeout will always happen before the default of the lender.

The interesting case where the two formulations disagree also in the co-monotonic case is when $\lambda_{B}<\lambda_{A}$, and hence $\tau^{B}>\tau^{A}$. In this case one can see by looking at (15) and (13) that

$$
V_{A}(t)=e^{-\int_{t}^{T} r(s) d s},
$$

which makes sense, given that default of $B$ will never happen to a solvent $A$, while the substitution closeout formula remains (13),

$$
\hat{V}_{A}(t)=e^{-\int_{t}^{T} r(s) d s}\left[\mathbb{Q}_{t}\left(\tau^{B}>T\right)+R^{B} \mathbb{Q}_{t}\left(\tau^{B} \leq T\right)\right],
$$

although we have to take into account Remark 1 if $t>0$.

\subsection{What happens in case of default. An empirical example.}

\subsubsection{The independence case}

With this simple model we can test numerically the behaviour of the formula (31) or (16) with risk-free closeout. Set the risk-free rate at $r=3 \%$, and consider a bond with maturity 5 years. The price of the bond varies with the default risk of the borrower, as usual, and here also with the default risk of the lender, due to the risk-free closeout. In Figure 1 we show the price of the bond for intensities $\lambda_{\text {Lender }}, \lambda_{\text {Borrower }}$ going from zero to $100 \%$. We consider $R^{\text {Borrower }}=0$ so that the level of the intensity approximately coincides with the market CDS spread on the 5 year maturity. 


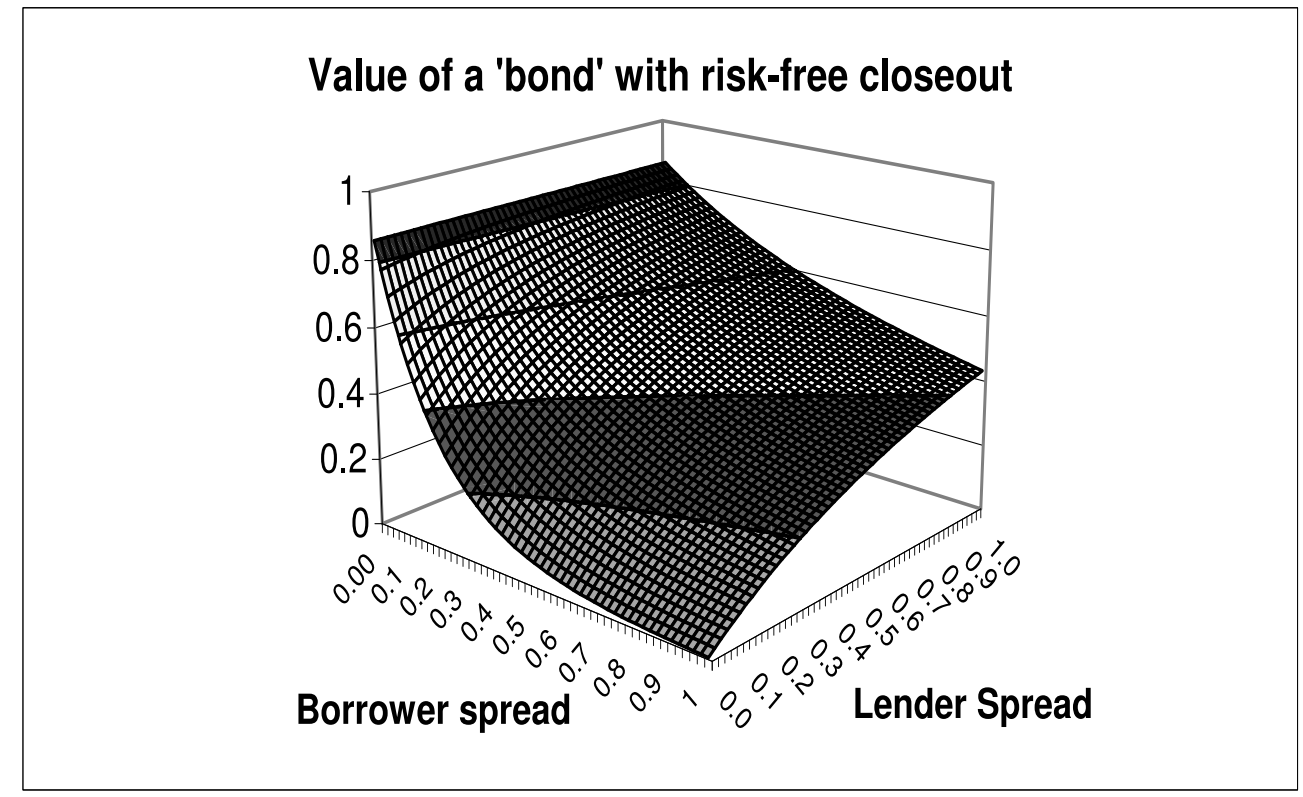

Figure 1

We see that the effect of the lender's risk of default is not negligible, and is particularly decisive when the borrower's risk is high. Market operators should be aware of this consequence of a risk-free closeout.

The results of Figure 1 can be compared with those of Figure 2, where we apply formula (10) or (13) that assumes a substitution closeout.

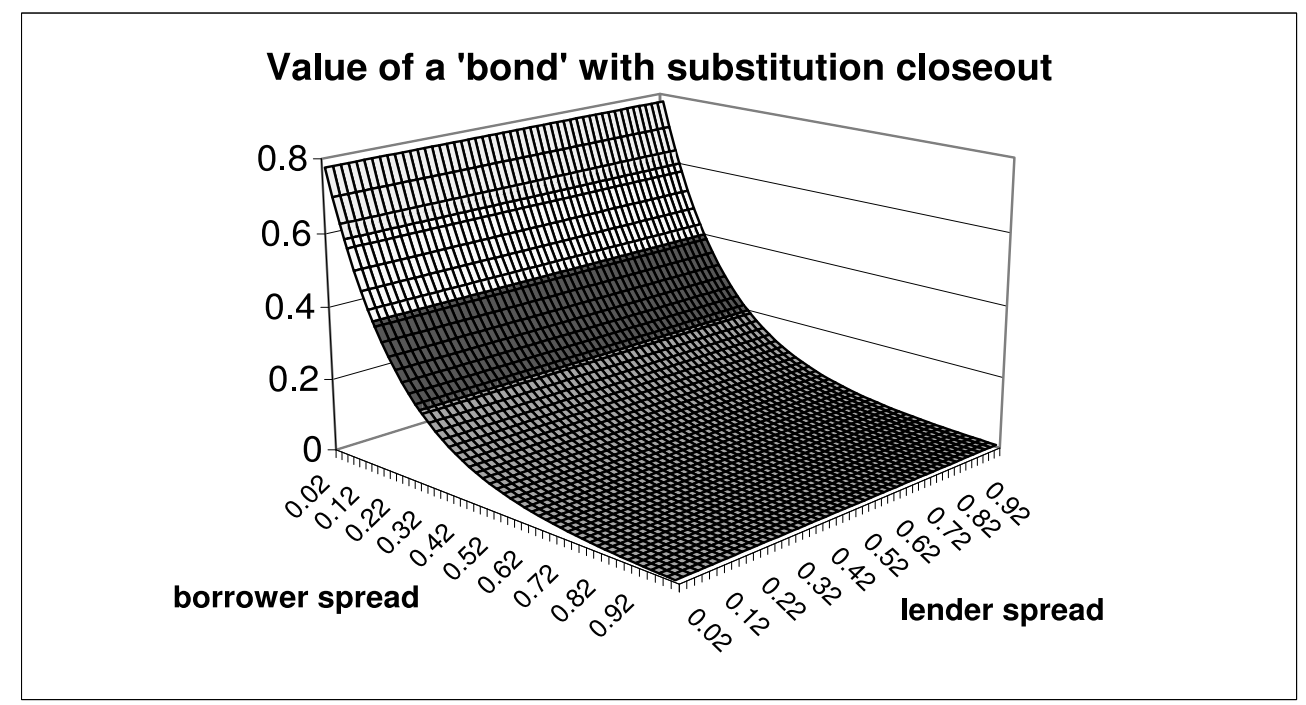

Figure 2

The pattern of Figure 2 traces precisely the pattern one would expect from standard financial principles: independence of the price of the deal from the risk of default of the counterparty which has no future obligations in the deal.

There is a further implication of a risk-free closeout that is at odds with market standards, and that should be pondered carefully by market operators and regulators. When bilateral 
counterparty risk adjustment is computed as indicated in the standard formula (3), that implies a risk-free closeout, we have a bizarre effect: a company which has a net creditor position in a derivative and suddenly defaults, can have a strong financial gain stemming from default itself, whose benefit will go to its liquidators.

Symmetrically, and this is the most worrying part, the counterparty that did not default and that is a net debtor of the defaulted company will have to book a sudden loss due to default of the creditor. Notice that this is at odds with standard financial wisdom. It is natural that a creditor of company is damaged by the default of the company, but here we have something more: when (3) is used, there is a damage not only to creditors, but also to debtors. This is worrying since it implies that with a risk-free closeout the default contagion spreads also to net debtors, not only to net creditors.

Let us observe a numerical example. We start from the above $r=3 \%$ and a bond with maturity 5 years, for a $1 \mathrm{bn}$ notional. Now we take $R^{\text {Borrower }}=20 \%$ and two risky parties. We suppose the borrower has a very low credit quality, as expressed by $\lambda_{\text {Borrower }}=0.2$, that means

$$
\mathbb{Q}\left(\tau^{\text {Borrower }} \leq 5 y\right)=63.2 \% .
$$

The lender has a much higher credit quality, as expressed by $\lambda_{\text {Lender }}=0.04$. This means a lower probability of default, that however is not negligible, being

$$
\mathbb{Q}\left(\tau^{\text {Lender }} \leq 5 y\right)=18.1 \% .
$$

A risk free bond issuance with the same maturity and notional would cost

$$
P_{T}=860.7 m n
$$

Using the formula with risk-free closeout, we get that a risky bond, within the two parties above, has price

$$
V_{\text {Lender }}=359.5 \mathrm{mn}
$$

to be compared with the price coming form the formula with substitution closeout

$$
\hat{V}_{\text {Lender }}=316.6 \mathrm{mn} \text {. }
$$

These figures can be very easily computed via (3) or more directly with (16). The example confirms a difference in the valuation given by the two formulas. The higher value of $V_{\text {Lender }}$ depends on the probability of default of the lender.

The difference of the two valuations is not negligible but not dramatic. More relevant is the difference of what happens in case of a default under the two assumptions on closeout. We have the following risk-adjusted probabilities on the happening of a default event

$$
\begin{aligned}
& \mathbb{Q}\left[\tau^{\text {Borrower }}<\min \left(5 y, \tau^{\text {Lender }}\right)\right]=58 \%, \\
& \mathbb{Q}\left[5 y<\min \left(\tau^{\text {Lender }}, \tau^{\text {Borrower }}\right)\right]=30 \%, \\
& \mathbb{Q}\left[\tau^{\text {Lender }}<\min \left(5 y, \tau^{\text {Borrower }}\right)\right]=12 \% .
\end{aligned}
$$

The two formulas agree on what happens in case of no default or in case of default of the borrower first. These are the most likely scenarios, totalling $88 \%$ probability. But with a non-negligible probability, $12 \%$, the lender can default first. Let us analyze in detail what happens in this case. Suppose the exact day when default happens is

$$
\tau^{\text {Lender }}=2.5 \text { years. }
$$

Just before default, at 2.5 years less one day, we have for the borrower the following book value of the liability produced by the above deal:

$$
V_{\text {Borrower }}\left(\tau^{\text {Lender }}-1 d\right)=-578.9 \mathrm{mln}
$$


if he assumes a risk-free closeout, or

$$
\hat{V}_{\text {Borrower }}\left(\tau^{\text {Lender }}-1 d\right)=-562.7 \mathrm{mln}
$$

if he assumes a substitution closeout.

Now default of the lender happens. In case of a risk-free closeout, one can easily check in (3) or (16), calling the lender $A$ and $B$ the borrower, that when $\tau^{A} \leq \min \left(T, \tau^{B}\right)$ and

$$
V_{B}^{0}\left(\tau^{A}\right) \leq 0,
$$

like here, we have

$$
V_{B}\left(\tau^{A}\right)=V_{B}^{0}\left(\tau^{A}\right) .
$$

Thus the book value of the bond becomes simply the value of a risk free bond,

$$
V_{B}\left(\tau^{A}+1 d\right)=-927.7 \mathrm{mln} .
$$

The borrower, which has not defaulted, must pay this amount entirely to the defaulted lender. He has a sudden loss of

$$
\begin{aligned}
& 927.7 \mathrm{mln}-578.9 \mathrm{mln} \\
= & 348.8 \mathrm{mln}
\end{aligned}
$$

due to default of the lender. This way of regulating bilateral counterparty risk leads to a default contagion that extends not only to the creditors, but also, and in a more sudden way, to the debtors of the defaulted company. This is a worrying effect that regulators can hardly consider desirable. Additionally, formulas (3) and (16) show clearly that the loss is higher the higher the credit spreads, and this is what makes this kind of contagion particularly dangerous. Finally, notice that this book loss of $365 \mathrm{mln}$ will soon become a material, realized loss because the borrower has to pay it to the liquidators of the defaulted lender, well before the original maturity of the deal.

Furthermore, this behaviour is not consistent with financial practice in the bond and loan market, where there are no big losses to the borrower or bond issuer due to the default of a lender or a bond-holder. This makes the behaviour particularly undesirable. We mentioned at the beginning of this section that with bilateral counterparty risk banks can book a profit when their own risk of default increases, and that this somewhat disturbing fact has been accepted also because it is consistent with the fair value option in the booking of bonds. Here we have found another disturbing effect when a risk-free closeout is considered: a sudden and big gain for the lender's liquidators when the lender himself defaults, and a symmetric big loss for the borrower. This is even more disturbing, and it is not even consistent with market practice on bonds.

The bond practice is instead consistent with the substitution closeout. In this case, applying (10) we have

$$
\hat{V}_{\text {Borrower }}\left(\tau^{\text {Lender }}+1 d\right)=-578.9 \mathrm{mln} .
$$

There is no discontinuity and no loss for the borrower in case of default of the lender.

We point out that the above example could be extended easily to those more complex derivative payoffs where there are, like in the 'derivative' bond, a clear lender and a clear borrower that do not change through the life of the deal. A party $X$ is a lender when $V_{X}^{0}(t)>0$, $T>t>0$, while he is a borrower if $V_{X}^{0}(t)<0, T>t>0$. Payoffs that generate this situation are very common. The simplest example is an option contract. The option buyer is the lender, the option writer is the borrower.

Additionally, some time after inception all deals, even the most complex derivatives, can have mark-to-market far away from zero, so that for one party $V_{X}^{0}(t) \ll 0$. Such party becomes a net borrower and in case of default of the other party would suffer, following a risk-free closeout, losses similar to those outlined above. 


\subsubsection{The co-monotonic case}

We now take an example where the two default times have constant intensities and are comonotonic, and we will see, conversely, that this time the substitution closeout has a dramatic effect for the creditors and liquidators of the defaulted company.

We assume $\lambda_{\text {Borrower }}=3.6 \%, \lambda_{\text {Lender }}=4 \%, r=3 \%, T=5 y$ and $R_{\text {Borrower }}=0$. This setting means that Lender always defaults first, since following formula (17)

$$
\tau^{\text {Borrower }}=\frac{\lambda_{\text {Lender }}}{\lambda_{\text {Borrower }}} \tau^{\text {Lender }}=1.11 \tau^{\text {Lender }}
$$

We still assume a notional of $1 \mathrm{bn}$. The initial value to the borrower of the bond under bilateral counterparty risk for the risk free closeout is

$$
-e^{-0.03 \cdot 5} 1 b n=-860.71 m n,
$$

namely the risk-free value, consistently with the example we showed in Section 6.1.5. The value of the bond under substitution closeout is

$$
-e^{-(0.066) \cdot 5} 1 b n=-718.92 m n
$$

Now let us see what happens when the lender A defaults at 2.5y. We need to remember that we are in a situation where $\mathrm{A}$ defaults always before $\mathrm{B}$, and where we know exactly that, if $\mathrm{A}$ defaults at $2.5 y$, B defaults in $2.77 y$. This is clearly a purely toy case, but it approximates a possibly realistic one: the case of a company B that, as an effect of the default of A, sees its own risk of default increase dramatically, going to the verge of default.

In such a case the risk free closeout gives us the same value both immediately before and after default, namely, to the lender A:

$$
e^{-0.03 \cdot 2.5} 1 b n=927.74 m n
$$

with no discontinuity, whereas the substitution closeout implies a jump from

$$
\begin{aligned}
& e^{-0.03 \cdot 2.5} \mathbb{Q}\left(\tau^{\text {Borrower }}>5 y \mid \tau^{\text {Lender }}>2.5 y\right) 1 b n \\
= & e^{-0.03 \cdot 2.5} \mathbb{Q}\left(\tau^{\text {Lender }}>\frac{5}{1.1} y \mid \tau^{\text {Lender }}>2.5 y\right) 1 b n \\
= & e^{-0.03 \cdot 2.5} e^{-0.04\left(\frac{5}{1.1}-2.5\right)}=856.41 b n
\end{aligned}
$$

to

$$
e^{-0.03 \cdot 2.5} \mathbb{Q}\left(\tau^{\text {Borrower }}>5 y \mid \tau^{\text {Lender }}=2.5 y\right) 1 b n=0 .
$$

Due to the fact that the model implies that the borrower will default at $2.77 \mathrm{y}$, which is before the maturity, the substitution closeout, that takes into account the default risk of the counterparty, sees a null value for the bond.

In other terms, at the default of the lender, the creditors and liquidators of the lender see all their claims towards the borrower lose their value. This confirms that the substitution closeout reduces the claims of the creditors towards the debtors of the defaulted entity, and this effect is stronger the stronger is the default dependence between the defaulted entity and its borrowers. This may be the case, for example, when the defaulted entity is a company with a relevant systemic impact.

\section{The collateral}

There is one final thing to assess, and it is the behaviour of the two forms of closeout for collateralized deals. A defaultable deal should satisfy the following property: the combination of the defaultable deal and a collateral agreement must eliminate losses at default. 
In a standardized collateral agrement for a deal with payoff $X$ the counterparty which is a net borrower provides the net lender with an amount of liquidity equal to the risk-free net present value of $X$. This amount of collateral generates interest and is regularly updated to remain equal to the net present value of $X$, and can be claimed back by the borrower only at maturity of the deal if the borrower has not defaulted earlier. If instead the borrower defaults earlier, the collateral must be used to offset the default loss.

We analyze in detail what happens at default considering a very simple payoff where $X=1$ and must be paid at $T$, namely the simple 'derivative' bond considered above. The borrower must give at time 0 to the lender an amount of liquidity equal to the risk-free net present value of the future payoff,

$$
C(0)=e^{-\int_{0}^{T} r(s) d s} .
$$

Then the borrower must update regularly this quantity, in such a way that the collateral remains equal at any time $t$ to the net present value of the future payoff,

$$
C(t)=e^{-\int_{t}^{T} r(s) d s} .
$$

The collateral is updated daily. We approximate this daily settlement with a continuous settlement. In this case, in order to keep the collateral $C(t)$ at $e^{-\int_{t}^{T} r(s) d s}$, the borrower must pay to the lender continuously an amount

$$
r(t) C(t) d t
$$

in fact it is trivial to show that if

$$
\begin{aligned}
d C(t) & =r(t) C(t) d t \\
C(0) & =e^{-\int_{0}^{T} r(s) d s}
\end{aligned}
$$

then

$$
C(t)=e^{-\int_{t}^{T} r(s) d s},
$$

as desired. On the other hand, the lender that keeps the collateral $C(t)$ must give back to the borrower the interest generated continuously by $C(t)$. This transforms into a continuous payment

$$
r(t) C(t) d t
$$

made by the lender to the borrower.

We immediately notice that the settlement payment (19) made by the borrower cancels out with the interest payment (20) made by the lender. Thus for a simple payoff the collateral agreement becomes a sort of self-financing strategy, namely it does not involve any net continuous exchange of money. The borrower $B$ pays $e^{-\int_{0}^{T} r(s) d s}$ at time 0 . The collateral remains with the lender and earns interest in time at rate $r(t)$, this interest $r(t) C(t) d t$ is not returned to the borrower but added to the value of the collateral, that in this way is always

$$
C(t)=e^{-\int_{t}^{T} r(s) d s} .
$$

\subsection{Risk-free closeout and collateral}

If we consider the risk-free closeout, the requirement of a match between the closeout and the value of the collateral at the first default $\tau$ among those of the two counterparties appears trivially obtained. The risk-free closeout amount is always

$$
V_{A}(\tau)=e^{-\int_{\tau}^{T} r(s) d s} .
$$


The value of the collateral at $\tau$ is always

$$
C(\tau)=e^{-\int_{\tau}^{T} r(s) d s} .
$$

If we just say that the collateral is an amount of money to be used to offset the default closeout, it turns out that the lender always has the right amount of money to offset the default closeout.

\subsection{Substitution closeout and collateral}

However, if we take a less naive view we remember that collateral is not just an amount of money but a contract with specific features. This contract has a value at default and it is this value, not the amount of money held by the lender, that needs to match the closeout amount. If this happens, there will be no transfer on money at default of a collateralized party and no losses for any party, exactly as we expect for a collateralized deal. We show in the following that this can be obtained rather easily with a substitution closeout. If we think of the practical working of collateral, we can treat collateral as an amount of money given to the lender from the borrower coupled with a claim of the borrower towards the lender to have this collateral back. If there is no default of the borrower before maturity, the borrower has a claim to have the collateral back at termination of the deal, namely a payoff $C(T)$ at maturity $T$ upon no default:

$$
1_{\left\{\tau^{B}>T\right\}} C(T)=1_{\left\{\tau^{B}>T\right\}} \quad \text { at } T .
$$

If instead the default of the borrower happens before maturity, the borrower has a claim to receive the collateral back at its own default time $\tau^{B}$

$$
1_{\left\{\tau^{B}<T\right\}} C(\tau)=1_{\left\{\tau^{B}<T\right\}} e^{-\int_{\tau^{B}}^{T} r(s) d s} \quad \text { at } \tau^{B} .
$$

In this simple setting we can easily check what happens when there is a default, either of the borrower or of the lender. In case the borrower defaults first, the closeout amount that the borrower should give to the lender is

$$
e^{-\int_{\tau B}^{T} r(s) d s}
$$

At the same time the borrower has the right to receive back the collateral, which has a value

$$
e^{-\int_{\tau B}^{T} r(s) d s}
$$

There is a perfect netting and a perfect match of collateral value and closeout amount of the deal at default. No money is transferred and no loss is suffered, as it should be for collateralized deals. This holds no matter whether we consider risk-free or substitution closeout, since we are considering the default of the borrower. At default of the borrower the residual net present value is risk free under either closeout.

More interesting is the case of the default of the lender at $\tau^{L} \leq T, \tau^{B}$. The borrower has a claim to receive back the collateral at maturity if the borrower itself does not default. The discounted expected value of such a claim is

$$
\mathbb{E}_{\tau^{L}}\left[e^{-\int_{\tau^{L}}^{T} r(s) d s} 1_{\left\{\tau^{B}>T\right\}}\right] .
$$

The claim of the lender depends on the choice about the closeout amount. In case of substitution closeout, the value of the lender's claim is the discounted expected payoff of the deal taking into account the possibility that $\tau^{B}$ defaults in the future,

$$
\mathbb{E}_{\tau^{L}}\left[e^{-\int_{\tau^{L}}^{T} r(s) d s} 1_{\left\{\tau^{B}>T\right\}}\right] .
$$

Again there is a perfect netting and a perfect match of collateral value and closeout amount of the deal at default. No money is transferred and no loss is suffered, as it should be for collateralized deals. When no default happens before maturity, the lender gives the collateral back to the borrower, again consistently with the practical working of collateral. 


\section{Conclusions}

In this paper we have analyzed the effect of the assumptions about the computation of the closeout amount on the counterparty risk adjustments of derivatives. We have compared the risk-free closeout assumed in the earlier literature with the substitution closeout we introduce here, which is inspired by the financial rationale contained in the recent ISDA documentation on the subject.

We have provided a formula for bilateral counterparty risk when a substitution closeout is used at default. We have reckoned that the substitution closeout is consistent with counterparty risk adjustments for standard and consolidated financial products such as bonds and loans. On the contrary the risk-free closeout introduces at time 0 a dependence on the risk of default of the party with no future obligations.

We have also shown that in case of risk-free closeout a party that is a net debtor of a company will have a sudden loss at the default of the latter, and this loss is higher the higher the debtor's credit spreads. This does not happen when a substitution closeout is considered.

Thus, the risk-free closeout increases the number of operators subject to contagion from a default, including parties that currently seem not to think they are exposed, and this is certainly a negative fact. On the other hand, it spreads the default losses on higher number of parties and reduces the classic contagion channel affecting creditors. For the creditors, this is a positive fact because it brings more money to the liquidators of the defaulted company. Additionally, a risk-free closeout is easier to implement at default time, since it corresponds to the net present value of a collateralized version of the deal. Thanks to this, one can also find it easier to understand why the provision of collateral makes a defaultable deal to become default-free. In the end, however, we have shown why if we take into account the contractual features of a collateral agreement, the provision of collateral makes a deal default free also when substitution closeout applies.

We think that the closeout issue should be considered carefully by market operators and regulators. For example, if the risk-free closeout introduced in the previous literature had to be recognized as a standard, banks should understand the consequences of this. In fact banks usually perform stress tests and set aside reserves for the risk of default of their net borrowers, but do not consider any risk related to the default of net lenders. The above computations, and the numerical examples, show that, should a risk-free closeout prevail, banks had better set aside important reserves against this risk. On the other hand, under substitution closeout, banks can expect the recovery to be lowered when their net borrowers default, compared to the case when a risk free closeout applies. In case of a substitution closeout, in fact, the money collected by liquidators from the counterparties will be lower, since deflated by the default probability of the counterparties themselves, and this will be even more evident if they are strongly correlated to the defaulted entity. 


\section{Appendix}

First of all, notice that from formula (10) one can compute that

$$
\begin{aligned}
\hat{V}_{B}(t)= & \mathbb{E}_{t}\left\{1_{0} \Pi_{B}(t, T)\right\} \\
& +\mathbb{E}_{t}\left\{1_{B}\left[\Pi_{B}\left(t, \tau^{B}\right)+D\left(t, \tau^{B}\right)\left(\left(V_{B}^{A}\left(\tau^{B}\right)\right)^{+}-R^{B}\left(-V_{B}^{A}\left(\tau^{B}\right)\right)^{+}\right)\right]\right\} \\
& +\mathbb{E}_{t}\left\{1_{A}\left[\Pi_{B}\left(t, \tau^{A}\right)+D\left(t, \tau^{A}\right)\left(R^{A}\left(V_{B}^{B}\left(\tau^{A}\right)\right)^{+}-\left(-V_{B}^{B}\left(\tau^{A}\right)\right)^{+}\right)\right]\right\} \\
= & -\mathbb{E}_{t}\left\{1_{0} \Pi_{A}(t, T)\right\} \\
& +\mathbb{E}_{t}\left\{1_{B}\left[-\Pi_{A}\left(t, \tau^{B}\right)+D\left(t, \tau^{B}\right)\left(\left(-V_{A}^{A}\left(\tau^{B}\right)\right)^{+}-R^{B}\left(V_{A}^{A}\left(\tau^{B}\right)\right)^{+}\right)\right]\right\} \\
& +\mathbb{E}_{t}\left\{1_{A}\left[-\Pi_{A}\left(t, \tau^{A}\right)+D\left(t, \tau^{A}\right)\left(R^{A}\left(-V_{A}^{B}\left(\tau^{A}\right)\right)^{+}-\left(V_{A}^{B}\left(\tau^{A}\right)\right)^{+}\right)\right]\right\} \\
= & -\hat{V}_{A}(t)
\end{aligned}
$$

so we have the desired symmetry property, like in the standard formula (3).

The standard formula (3) with risk-free closeout can be simplified to

$$
\begin{aligned}
& \hat{V}_{A}(t)=V_{A}^{0}(t) \\
& +\mathbb{E}_{t}\left[L^{A} 1_{A} D\left(t, \tau^{A}\right) \cdot\left(-V_{A}^{0}\left(\tau^{A}\right)\right)^{+}\right] \\
& -\mathbb{E}_{t}\left[L^{B} 1_{B} D\left(t, \tau^{B}\right) \cdot\left(V_{A}^{0}\left(\tau^{B}\right)\right)^{+}\right] .
\end{aligned}
$$

The alternative formula (10) with replacement-cost closeout does not allow such a trivial simplification. However some simplifications are possible by replacing $V_{A}^{B}\left(\tau^{1}\right)$ and $V_{A}^{A}\left(\tau^{B}\right)$ with their definitions, obtaining

$$
\begin{aligned}
& \hat{V}_{A}(t)=\mathbb{E}_{t}\left\{1_{0} \Pi_{A}(t, T)\right\} \\
& +\mathbb{E}_{t}\left\{1 _ { A } \left[\Pi_{A}\left(t, \tau^{A}\right)+D\left(t, \tau^{A}\right)\left(\left(V_{A}^{0}\left(\tau^{A}\right)-\mathbb{E}_{\tau^{A}}\left[L^{B} 1_{\left\{\tau^{B} \leq T\right\}} D\left(\tau^{A}, \tau^{B}\right) \cdot\left(V_{A}^{0}\left(\tau^{B}\right)\right)^{+}\right]\right)^{+}\right.\right.\right. \\
& \left.\left.\left.-R^{A}\left(-V_{A}^{0}\left(\tau^{A}\right)+\mathbb{E}_{\tau^{A}}\left[L^{B} 1_{\left\{\tau^{B} \leq T\right\}} D\left(\tau^{A}, \tau^{B}\right) \cdot\left(V_{A}^{0}\left(\tau^{B}\right)\right)^{+}\right]\right)^{+}\right)\right]\right\} \\
& +\mathbb{E}_{t}\left\{1 _ { B } \left[\Pi_{A}\left(t, \tau^{B}\right)+D\left(t, \tau^{B}\right)\left(R^{B}\left(V_{A}^{0}\left(\tau^{B}\right)+\mathbb{E}_{\tau^{B}}\left[L^{A} 1_{\left\{\tau^{A} \leq T\right\}} D\left(\tau^{B}, \tau^{A}\right) \cdot\left(-V_{A}^{0}\left(\tau^{A}\right)\right)^{+}\right]\right)^{+}\right.\right.\right. \\
& \left.\left.\left.-\left(-V_{A}^{0}\left(\tau^{B}\right)-\mathbb{E}_{\tau^{B}}\left[L^{A} 1_{\left\{\tau^{A} \leq T\right\}} D\left(\tau^{B}, \tau^{A}\right) \cdot\left(-V_{A}^{0}\left(\tau^{A}\right)\right)^{+}\right]\right)^{+}\right)\right]\right\} .
\end{aligned}
$$

If we replace

$$
1_{0}=1-1_{A}-1_{B}
$$

recalling

$$
\Pi_{A}(t, \tau)-\Pi_{A}(t, T)=-D(t, \tau) \Pi_{A}(\tau, T)
$$


we obtain

$$
\begin{aligned}
& \hat{V}_{A}(t)=\mathbb{E}_{t}\left\{\Pi_{A}(t, T)\right\} \\
& +\mathbb{E}_{t}\left\{1 _ { A } \left[-D\left(t, \tau^{A}\right) \Pi_{A}\left(\tau^{A}, T\right)+D\left(t, \tau^{A}\right)\left(\left(V_{A}^{0}\left(\tau^{A}\right)-\mathbb{E}_{\tau^{A}}\left[L^{B} 1_{\left\{\tau^{B} \leq T\right\}} D\left(\tau^{A}, \tau^{B}\right) \cdot\left(V_{A}^{0}\left(\tau^{B}\right)\right)^{+}\right]\right)^{+}\right.\right.\right. \\
& \left.\left.\left.-R^{A}\left(-V_{A}^{0}\left(\tau^{A}\right)+\mathbb{E}_{\tau^{A}}\left[L^{B} 1_{\left\{\tau^{B} \leq T\right\}} D\left(\tau^{A}, \tau^{B}\right) \cdot\left(V_{A}^{0}\left(\tau^{B}\right)\right)^{+}\right]\right)^{+}\right)\right]\right\} \\
& +\mathbb{E}_{t}\left\{1 _ { B } \left[-D\left(t, \tau^{B}\right) \Pi_{A}\left(\tau^{B}, T\right)+D\left(t, \tau^{B}\right)\left(R^{B}\left(V_{A}^{0}\left(\tau^{B}\right)+\mathbb{E}_{\tau^{B}}\left[L^{A} 1_{\left\{\tau^{A} \leq T\right\}} D\left(\tau^{B}, \tau^{A}\right) \cdot\left(-V_{A}^{0}\left(\tau^{A}\right)\right)^{+}\right]\right)^{+}\right.\right.\right. \\
& \left.\left.\left.-\left(-V_{A}^{0}\left(\tau^{B}\right)-\mathbb{E}_{\tau^{B}}\left[L^{A} 1_{\left\{\tau^{A} \leq T\right\}} D\left(\tau^{B}, \tau^{A}\right) \cdot\left(-V_{A}^{0}\left(\tau^{A}\right)\right)^{+}\right]\right)^{+}\right)\right]\right\}
\end{aligned}
$$

Now we use

$$
(X)^{+}-R(-X)^{+}=X+(1-R)(-X)^{+}
$$

and

$$
R(X)^{+}-(-X)^{+}=X-(1-R)(X)^{+}
$$

getting

$$
\begin{aligned}
& \hat{V}_{A}(t)=\mathbb{E}_{t}\left\{\Pi_{A}(t, T)\right\} \\
& +\mathbb{E}_{t}\left\{1 _ { A } \left[-D\left(t, \tau^{A}\right) \Pi_{A}\left(\tau^{A}, T\right)+D\left(t, \tau^{A}\right)\left(V_{A}^{0}\left(\tau^{A}\right)-\mathbb{E}_{\tau^{A}}\left[L^{B} 1_{\left\{\tau^{B} \leq T\right\}} D\left(\tau^{A}, \tau^{B}\right) \cdot\left(V_{A}^{0}\left(\tau^{B}\right)\right)^{+}\right]\right.\right.\right. \\
& \left.\left.\left.+\left(1-R^{A}\right)\left(-V_{A}^{0}\left(\tau^{A}\right)+\mathbb{E}_{\tau^{A}}\left[L^{B} 1_{\left\{\tau^{B} \leq T\right\}} D\left(\tau^{A}, \tau^{B}\right) \cdot\left(V_{A}^{0}\left(\tau^{B}\right)\right)^{+}\right]\right)^{+}\right)\right]\right\} \\
& +\mathbb{E}_{t}\left\{1 _ { 2 } \left[-D\left(t, \tau^{B}\right) \Pi_{A}\left(\tau^{B}, T\right)+D\left(t, \tau^{B}\right)\left(V_{A}^{0}\left(\tau^{B}\right)+\mathbb{E}_{\tau^{B}}\left[L^{A} 1_{\left\{\tau^{A} \leq T\right\}} D\left(\tau^{B}, \tau^{A}\right) \cdot\left(-V_{A}^{0}\left(\tau^{A}\right)\right)^{+}\right]\right.\right.\right. \\
& \left.\left.\left.-\left(1-R^{B}\right)\left(V_{A}^{0}\left(\tau^{B}\right)+\mathbb{E}_{\tau^{B}}\left[L^{A} 1_{\left\{\tau^{A} \leq T\right\}} D\left(\tau^{B}, \tau^{A}\right) \cdot\left(-V_{A}^{0}\left(\tau^{A}\right)\right)^{+}\right]\right)^{+}\right)\right]\right\}
\end{aligned}
$$

Now we apply the law of iterated expectation, exploiting

$$
\begin{aligned}
& \mathbb{E}_{t}\left[-D(t, \tau) \Pi_{A}(\tau, T)+D(t, \tau) V_{A}^{0}(\tau)\right] \\
= & \mathbb{E}_{t}\left[-D(t, \tau) \Pi_{A}(\tau, T)+D(t, \tau) \mathbb{E}_{\tau}\left[\Pi_{A}(\tau, T)\right]\right] \\
= & \mathbb{E}_{t}\left[-D(t, \tau) \mathbb{E}_{\tau}\left[\Pi_{A}(\tau, T)\right]+D(t, \tau) \mathbb{E}_{\tau}\left[\Pi_{A}(\tau, T)\right]\right]=0,
\end{aligned}
$$

so that

$$
\begin{aligned}
& \hat{V}_{A}(t)=\mathbb{E}_{t}\left\{\Pi_{A}(t, T)\right\} \\
& +\mathbb{E}_{t}\left\{1 _ { 1 } \left[D ( t , \tau ^ { A } ) \left(-\mathbb{E}_{\tau^{A}}\left[L^{B} 1_{\left\{\tau^{B} \leq T\right\}} D\left(\tau^{A}, \tau^{B}\right) \cdot\left(V_{A}^{0}\left(\tau^{B}\right)\right)^{+}\right]\right.\right.\right. \\
& \left.\left.\left.+\left(1-R^{A}\right)\left(-V_{A}^{0}\left(\tau^{A}\right)+\mathbb{E}_{\tau^{A}}\left[L^{B} 1_{\left\{\tau^{B} \leq T\right\}} D\left(\tau^{A}, \tau^{B}\right) \cdot\left(V_{A}^{0}\left(\tau^{B}\right)\right)^{+}\right]\right)^{+}\right)\right]\right\} \\
& +\mathbb{E}_{t}\left\{1 _ { 2 } \left[D ( t , \tau ^ { B } ) \left(\mathbb{E}_{\tau^{B}}\left[L^{A} 1_{\left\{\tau^{A} \leq T\right\}} D\left(\tau^{B}, \tau^{A}\right) \cdot\left(-V_{A}^{0}\left(\tau^{A}\right)\right)^{+}\right]\right.\right.\right. \\
& \left.\left.\left.-\left(1-R^{B}\right)\left(V_{A}^{0}\left(\tau^{B}\right)+\mathbb{E}_{\tau^{B}}\left[L^{A} 1_{\left\{\tau^{A} \leq T\right\}} D\left(\tau^{B}, \tau^{A}\right) \cdot\left(-V_{A}^{0}\left(\tau^{A}\right)\right)^{+}\right]\right)^{+}\right)\right]\right\} .
\end{aligned}
$$




\section{References}

[1] Bielecki, T., and Rutkowski M. (2001). Credit risk: Modeling, Valuation and Hedging. Springer Verlag.

[2] Black, F. and Cox, J. C. (1976). Valuing corporate securities: some effects of bond indenture provisions. Journal of Finance. 31:351-367

[3] D. Brigo, I. Bakkar. Accurate counterparty risk valuation for energy-commodities swaps. Energy Risk. March 2009 issue.

[4] Brigo, D. and Capponi, A. (2008). Bilateral counterparty risk valuation with stochastic dynamical models and application to CDSs. Working paper available at http://arxiv.org/abs/0812.3705. Short updated version in Risk Magazine, March 2010.

[5] D. Brigo and K. Chourdakis (2009). Counterparty Risk for Credit Default Swaps: Impact of spread volatility and default correlation. International Journal of Theoretical and Applied Finance, Vol. 12 (07), pages 1007-1026..

[6] D. Brigo and M. Masetti. Risk Neutral Pricing of Counterparty Risk. In: Pykhtin, M. (Editor), Counterparty Credit Risk Modeling: Risk Management, Pricing and Regulation. Risk Books, 2005, London.

[7] Brigo, D., Pallavicini, A., and V. Papatheodorou (2010) Bilateral counterparty risk valuation for interest-rate products: impact of volatilities and correlations. working paper avaialable at http://papers.ssrn.com/sol3/papers.cfm?abstract_id=1507845.

[8] J. K. Gregory (2009). "Being two faced over counterparty credit risk", Risk Magazine 22 (2), pages 86-90.

[9] ISDA - International Swaps and Derivatives Association, Inc. (2009). "ISDA CLOSEOUT AMOUNT PROTOCOL", available at www.isda.org/isdacloseoutamtprot/docs/isdacloseoutprot-text.pdf

[10] ISDA - International Swaps and Derivatives Association, Inc. (2010). Market Review of OTC Derivative Bilateral Collateralization Practices, March 1, 2010

[11] Marshall, A., and I. Olkin (1967). A multivariate exponential distribution. Journal of the American Statistical Association, Vol 62, No. 317, pp 30-44.

[12] Morini, M. and A. Prampolini (2010): Risky funding: a unified framework for counterparty and liquidity charges, available at http://ssrn.com/abstract $=1506046$.

[13] Morini, M. One more Model Risk when using gaussian copula for Risk Management, available at http://ssrn.com/abstract $=1520670$.

[14] E. Picoult (2005). Calculating and Hedging Exposure, Credit Value Adjustment and Economic Capital for Counterparty Credit Risk, in "Counterparty Credit Risk Modelling" (M. Pykhtin, ed.), Risk Books. 